\title{
The Christmas Island blue-tailed skink (Cryptoblepharus egeriae): a survey protocol and an assessment of factors that relate to occupancy and detection
}

\author{
Michael J. Smith, Christopher R.J. Boland, Dion Maple and Brendan Tiernan
}

Parks Australia, Christmas Island National Park, Christmas Island, Western Australia 6798, Australia, Indian Ocean. Email: myobatrachid@hotmail.com

\begin{abstract}
The endemic Christmas Island blue-tailed skink (Cryptoblepharus egeriae) is in serious decline and only one known population remains on Christmas Island, despite it being widespread and common up until the 1990s. Here, we examine the relationship between occupancy, detectability, and several key environmental predictors and describe a passive observation survey protocol for the species that incorporates detection probabilities. By incorporating several covariates on detection and occupancy that we, a priori, considered to be important, we found $C$. egeriae was more likely to be found in habitat with an intermediate canopy cover. Detection probability using our survey protocol (0.28 with $95 \% \mathrm{Cl}: 0.17$ to 0.43 ) was weakly but positively related to survey hour (10:00 to 14:00 local time), indicating afternoons were a better time to survey. Based upon a value of 0.28 , we predict that four to five survey visits over a reasonably short period of time ( $\approx$ one month) will sufficiently minimise the likelihood of recording a false absence for the species.
\end{abstract}

KEYWORDS: Christmas Island, blue-tailed skink, Cryptoblepharus egeriae, survey protocol, occupancy, detection

\section{INTRODUCTION}

Christmas Island (Figure 1) is situated in the Indian Ocean approximately $360 \mathrm{~km}$ south of the western head of Java, Indonesia (Claussen 2005). The island is around $135 \mathrm{~km}^{2}$ and table-topped with a central plateau (Claussen 2005). The area between the central plateau and the coastline comprises a series of steep limestone cliffs, terraces, and slopes (Claussen 2005) that are difficult to traverse. Most of the island is either National Park (c. $63 \%$ of the land area) or mine lease (approximately $25 \%$ of the island has been mined for phosphate) which create a mosaic of different habitats that range from deep soiled, intact forests with little to no understorey (O'Dowd and Lake 1990; O'Dowd and Lake 1991; Green 1997) through to mined and denuded pinnacle fields in different stages of vegetation succession.

The blue-tailed skink Cryptoblepharus egeriae (Boulenger, 1889) is an Indian Ocean Christmas Island endemic (Cogger et al. 1983). Despite being abundant in the early 1980s (Cogger et al. 1983), the species is now in serious decline with the last known population occurring on the southwestern end of the island (Egeria Point; Figure 1). To date, the causes of the declines are not fully understood, but the introduction of predators such as the yellow crazy ant (Anoplolepis gracilipes
Smith, 1857), giant centipede (Scolopendra subspinipes Leach, 1815), and Asian wolf snake (Lycodon capucinus Boie, 1827) are likely to have played a major role (unpublished data).

Declines in C. egeriae were first reported in the early 1990s as the skink was thought to be disappearing from the islands' settled area (north-eastern end of the island). This pattern of decline was confirmed by Cogger and Sadlier (1999). By early 2009, the species was only known to occur in the north-western and south-western tips of the island and by mid 2009 the only known population occurred on the south western tip of the island (unpublished data). Although there have been a number of surveys for C. egeriae since the $1980 \mathrm{~s}$, there is need for a consistent island-wide survey effort with a rigorous survey protocol. Additionally, Christmas Island National Park, in conjunction with Taronga Zoo, have undertaken a captive breeding program for $C$. egeriae with the ultimate goal of reintroduction into the wild. A basic requirement of any reintroduction program is an ability to not only assess success through an appropriate and quantifiable survey protocol, but to also ensure that the species was not present prior to reintroduction.

There has not been any attempt to devise an appropriate and consistent survey protocol for $C$. egeriae that incorporates imperfect detection (cf. 


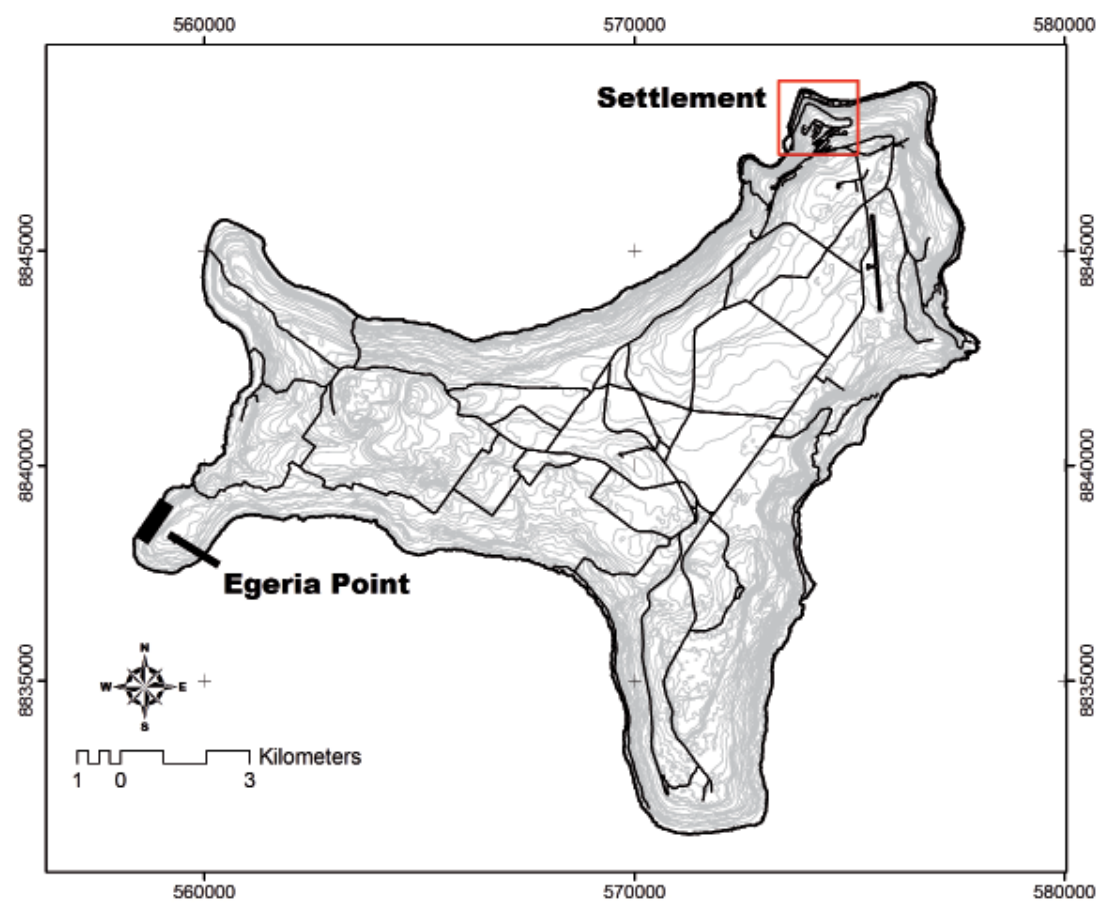

FIGURE 1 Christmas Island showing Egeria Point, the study area (black rectangle), $25 \mathrm{~m}$ contours (grey lines), roads (black lines), and the main settled area on the island (Settlement). Projection is UTM Zone 48 south (WGS84).

Royle and Dorazio 2008). Given the extent of declines in $C$. egeriae, developing a survey protocol that is optimal within the confines of the various limitations (both resources and physical conditions that are a feature of Christmas Island) is of critical importance if undiscovered populations are to be detected and given the possibility of future reintroduction efforts. One survey technique that has been commonly used for $C$. egeriae is to wait quietly in an area for a period of time (typically around 10 minutes) and count the number of individuals. We view this as a reasonable starting point for a species-specific survey protocol. Further, Christmas Island National Park (CINP) conducts a biennial island wide survey (IWS) for Gecarcoidea natalis Pocock, 1888 (red crabs) and A. gracilipes at around 900 points across the entire island. Incorporating surveys for $C$. egeriae into the IWS may help to provide a baseline data set for the management and conservation of the species.

Here, we outline and assess a survey protocol for C. egeriae that accounts for detectability and relates both occupancy and detectability to several potentially important environmental variables (cf. Royle and Dorazio 2008).

\section{MATERIALS AND METHODS}

Using GRASS (6.3.0; Neteler and Mitasova 2008) we positioned a $500 \mathrm{~m} \times 150 \mathrm{~m}$ grid consisting of $5 \mathrm{~m} \times 5$ $\mathrm{m}$ cells over an area of known occupancy by $C$. egeriae (Figure 1). Forty randomly chosen grid cells were then selected as survey sites for the skink (Figure 2). Each survey site was visited on three occasions between 12 and 27 March 2009 and consisted of a circle with a 5 $\mathrm{m}$ radius centred on the centre of the $5 \mathrm{~m} \times 5 \mathrm{~m}$ grid. The surveys were not conducted on rainy days; however there was the occasional brief rainy period during some surveys. On each visit, each site was surveyed for 10 minutes with two people facing in opposite directions so that they each quietly surveyed a non-overlapping $180^{\circ}$ field of view.

\section{THE COVARIATES}

In the past, surveyors have focused their survey effort in areas that met a preconceived notion that the lizards frequented forested areas with disturbed canopy and large fallen logs. With this in mind, we included two occupancy covariates, an estimate of the proportion of each study site covered by dead logs (or fallen trees that were not necessarily dead) that were greater than $5 \mathrm{~cm}$ in diameter and the proportion canopy cover. We did not include live standing trees. The entire study area consists of forests dominated by species such as Barringtonia racemosa, Scaevola taccada and Pandanus christmatensis. The proportion of canopy cover was calculated as the mean estimate of the proportion of the study site bathed in sunlight across the three visits. This produced an adequate estimate of canopy cover as all sites had estimates made during the middle of the day when the sun was at its highest. On each visit, the two surveyors agreed on the proportion of the site (circle with $5 \mathrm{~m}$ radius) bathed in sunlight and 


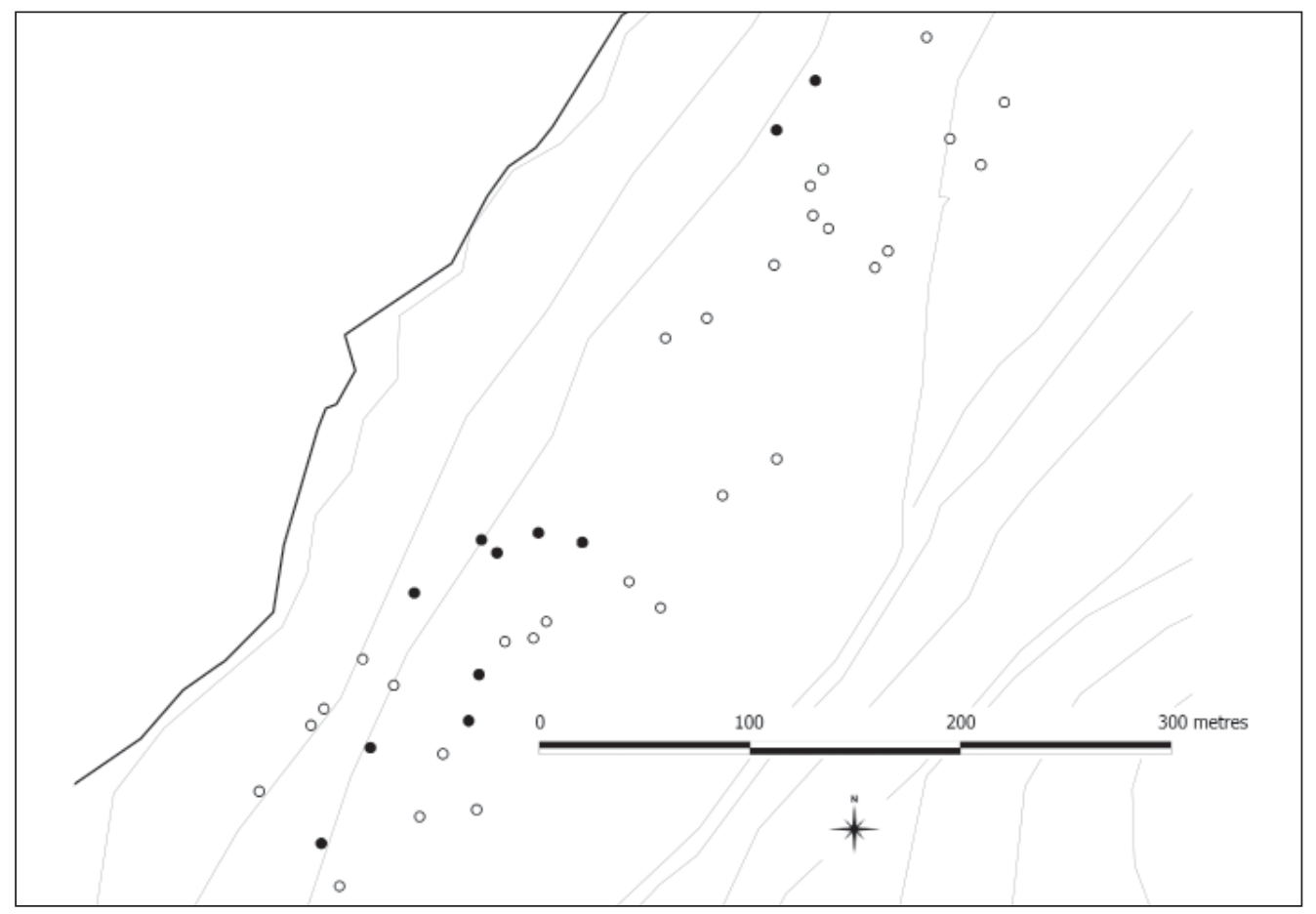

FIGURE 2 Distribution of the study sites at Egeria Point. Open circles indicate sites where Cryptoblepharus egeriae was not detected on any survey and solid circles indicate sites where $C$. egeriae was detected.

the proportion covered by $\operatorname{logs}>5 \mathrm{~cm}$ in diameter.

On each visit, we recorded the proportion of each 10 minute survey time that each site received direct sunlight (sunlight, for example, varied with cloud cover) and included this estimate as a covariate on detectability. We also recorded the start time of each survey, which ranged from 10:00 to 14:00 local time. The detectability covariates were also included because we had a priori reason to think that they may influence detectability. Temperature was not included as it was not greatly variable (mean $\pm \mathrm{SD}=30^{\circ} \mathrm{C} \pm 2{ }^{\circ} \mathrm{C}$ ), but did follow a typical circadian rhythm with highest temperatures reached between early and mid afternoon.

\section{STATISTICAL ANALYSIS}

We then generated statistical models to make inferences from repeated survey data regarding the underlying rate of occupancy, as well as the probability of detection at occupied sites (cf. Royle and Dorazio 2008). As it could not be assumed that $C$. egeriae would be detected on each visit to each site, it was necessary to account for imperfect detectability in the statistical analysis of the data. Accordingly, we treated the sites as being drawn from one of two discrete categories: occupied, where on each survey the species will be detected with an unknown probability, $p$, or unoccupied, where the species will not be detected, as it is absent (cf. MacKenzie et al., 2006; Royle and Dorazio 2008). By carrying out three repeated surveys at the sample sites, we were able to make statistical inferences regarding the actual rate of occupancy, allowing for the fact that the species in question may not have been detected at some sites that were actually occupied. The basic form of the occupancy model, where all sites have a common probability of occupancy, and all surveys have a common probability of detecting the species at occupied sites, can be extended through a logistic regression to relate the effects of covariates on both the occupancy and detection probabilities associated with sites and surveys.

We followed the Site-Occupancy Species Distribution Modelling approach outlined in Kéry (2010) whereby we used a nonstandard generalized linear mixed model (GLMM) to model both occupancy (state model) and detection (observation model) using a hierarchical, coupled logistic regression (refer to Kéry 2010 for a more detailed description of this modelling approach). The modelling was conducted within the freely-available Bayesian statistics package OpenBugs 2.2.0 which was run through $\mathrm{R}$ statistical software (R Development Core Team 2007) with the package BRugs.

The detection model takes the general form of: $\operatorname{logit}\left(p_{i j}\right)=\alpha_{0}+\alpha_{1}$. Time in $\operatorname{Sun}_{\mathrm{ij}}+\alpha_{2}$.Start Time $\mathrm{ij}_{\mathrm{ij}}$ and the occupancy model:

$\operatorname{logit}\left(\psi_{i}\right)=\beta_{0}+\beta_{1} \operatorname{Logs}_{\mathrm{i}}+\beta_{2}$. Canopy Cover $_{\mathrm{i}}+\beta_{3}$. Canopy Cover $_{\mathrm{i}}$. Canopy Cover . .

where $i$ and $j$ indicate site and visit respectively. We suspected that occupancy may be lowest at high and low canopy cover levels and consequently included a quadratic term for canopy cover.

Vague (uninformative) normally distributed ( 0 , 1.0E-3) priors were used for all model parameters and model sensitivity to priors was assessed by comparing 
the normal model predictions with those from a model with uniform $(-10,10)$ prior distributions (cf. Kéry 2010). The covariates were standardised by subtracting the mean and dividing by the standard deviation prior to analysis to improve model fit. Model goodness-of-fit was assessed by calculating a Bayesian p-value that was based upon the posterior predictive distributions of the discrepancy measures (Kéry 2010). Convergence of the MCMC algorithm was checked by examining the output of three replicate Markov chains with differing starting values, both by visual inspection of the outputs and by computing the Brooks-Gelman-Rubin convergence statistic. Final inferences were made by discarding the first 15,000 iterations and retaining the next 45,000 iterations for further inference and thinning by 25 iterations.

Finally, we used the equation:

$\mathrm{P}_{\mathrm{N}}=1-(1-p)^{\mathrm{N}}$

to determine the overall detection probability of the survey protocol relative to the number of visits, where $p=$ estimated detection probability and $\mathrm{N}=$ number of visits.

\section{RESULTS}

Specimens of Cryptoblepharus egeriae were detected in 11 of the 40 randomly chosen study sites (Figure 2). The Bayesian p-value for the model was 0.33 , indicating an adequate fit. We found support for several detection/ covariate or occupancy/covariate relationships (Table 1).

Detection was positively related to time bathed in sunlight and survey start time. However, the 95\% Credibility Intervals for time bathed in sunlight incorporated zero suggesting the relationship was not statistically defensible (Table 1). However, the 95\% Credibility Intervals for start time only just crossed zero indicating more support for that relationship (Table 1)

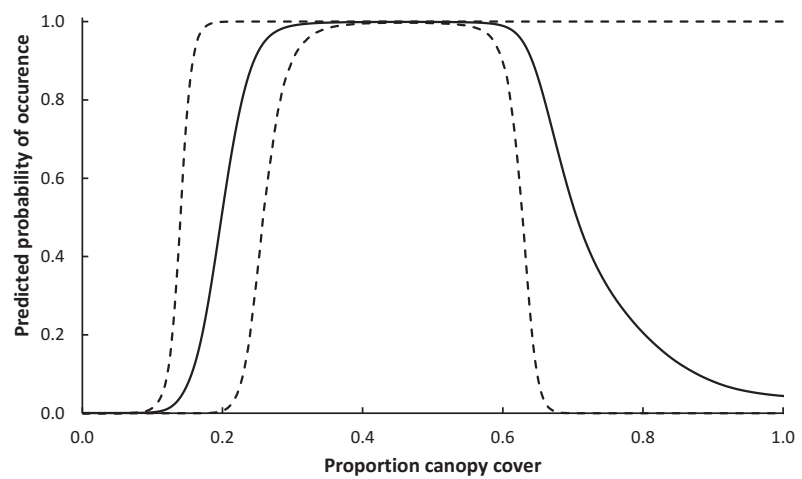

FIGURE 3 Predicted relationship between occupancy by Cryptoblepharus egeriae and proportion canopy cover. Solid line indicates posterior mean and dashed lines delineate $95 \% \mathrm{Cl}$.

and suggests that lizards may be more detectable when the sites are surveyed later in the day (up until 14:00 Eastern Standard Time). The mean detectability estimate was 0.28 (95\% Credibility Interval: 0.17 to 0.43 ).

Occupancy related positively and strongly to canopy cover and strongly and negatively to its quadratic term (95\% Credibility Intervals did not cross zero; Table 1). A positive main effect and negative quadratic term indicates that sites with intermediate canopy cover are favoured by the skinks. Our data suggests that sites with canopy covers from around $30 \%$ to $60 \%$ were preferred (Figure 3). We found no evidence that the presence of logs in the survey area related to occupancy.

Taking into account the environmental predictors and the detection probability of the survey protocol, our modelling predicted that around 20 (95\% Credibility Interval: 16 to 25$)$ sites were actually occupied. Based upon a detection probability of 0.28 , we estimate that it would take four to five visits to a site over a short period

TABLE 1 Posterior parameter estimates for occupancy $(\psi)$ and detection $(p)$. Sun = proportion of each 10-minute survey period that the site was bathed in sunlight, Start = survey start time, Log = proportion of survey area covered in dead logs $>5 \mathrm{~cm}$ in diameter, Canopy = proportion canopy cover, Canopy^2 = quadratic canopy cover term.

mean

$$
\text { sd }
$$

0.025
0.5

11.39

$-2.06$

16.45

$-13.83$

$\begin{array}{ll}4.84 & 9.25 \\ -7.83 & -3.70 \\ 8.18 & 13.85 \\ -19.58 & -16.70\end{array}$

$-19.58$

2.52

2.52

Canopy $^{\wedge} 2$

Detection

\begin{tabular}{llllllll} 
Intercept & -1.35 & 0.39 & -2.15 & -1.60 & -1.33 & -1.09 & -0.61 \\
Sun & 0.69 & 0.53 & -0.29 & 0.33 & 0.66 & 1.01 & 1.83 \\
Start & 0.67 & 0.36 & -0.01 & 0.42 & 0.66 & 0.90 & 1.39 \\
\hline
\end{tabular}


of time (e.g. within one month) to be around $70 \%$ to $80 \%$ confident of true absence.

\section{DISCUSSION}

The decline in the distribution of the Christmas Island endemic skink Cryptoblepharus egeriae appears to be wide-ranging. However, Christmas Island features large areas of rugged landscapes that are difficult to access (Hicks et al. 1990) and accordingly, the possibility cannot be discounted that undiscovered populations still exist on the island. Consequently, an appropriate and quantifiable survey protocol is essential. Additionally, an appropriate survey protocol is a basic requisite to assess the success of any reintroduction attempts from captive bred stock, which are proposed for the island. Our results suggest that the passive survey technique outlined in this study is appropriate as long as four to five repeat surveys are conducted on sunny days within a short time period $(\approx$ one month) to sufficiently minimise the probability of recording a false absence.

In the study area, we also found that $C$. egeriae were more likely to occupy sites with an intermediate canopy cover. These conditions presumably provide a combination of sun and shade that best meets the specific ecological requirements of the species. Consequently, future surveys should attempt to incorporate areas with moderate canopy cover, typically ranging from around $30 \%$ to $60 \%$. Even though there has been a preconception that $C$. egeriae prefer habitats with dead logs, we were unable to substantiate that claim. In the randomly chosen sites, $C$. egeriae were just as likely to occupy habitats with few to no large logs. However, fallen trees can reduce canopy cover which may then create habitat with a moderate canopy cover that is more likely to be occupied by the species.

We believe that the geographical isolation of Egeria Point is an important consideration in the declines. The area that the skinks occupied at the time of the study is at least a 1.5 to $2.0 \mathrm{~km}$ walk across difficult habitat from the nearest road. Appropriately surveying other isolated habitats on the island will be an important step in trying to locate new populations. Using the survey technique outlined here should provide a means of quantifying the species distribution and likelihood of occupancy.

\section{ACKNOWLEDGEMENTS}

This research has been funded by the Australian Director of National Parks. We would like to acknowledge the people that have assisted in the field, which includes Rachel Barr, Fairuz Abdul Halim, Claire Humphries, Dylan Juhasz, Rebecca Reeves, Fraser Scott, Kent Retallick, and Penny Watson.

\section{REFERENCES}

Claussen, J. (2005). Native plants of Christmas Island. Flora of Australia Supplementary Series 22. Australian Government Department of the Environment and Heritage, Australian Biological Resources Study, Canberra.

Cogger, H., Sadlier, R., and Cameron, E., (1983). The terrestrial reptiles of Australia's island territories. Australian National Parks and Wildlife Service, Canberra. Special Edition No. 11.

Cogger, H. and Sadlier, R. (1999). The terrestrial reptiles of Christmas Island: a reappraisal of their status. The Australian Museum, Sydney.

Green, P.T. (1997). Red crabs in rain forest on Christmas Island, Indian Ocean: Activity patterns, density and biomass. Journal of Tropical Ecology 13(1): 17-38.

Hicks, J., Rumpff, H., and Yorkston, H. (1990). Christmas crabs. National Library of Australia, Canberra.

Kéry, M. (2010). Introduction to WinBUGS for ecologists. Academic Press, Amsterdam.

MacKenzie, D.I., Nichols, J.D., Royle, J.A., Pollock, K.H., Bailey, L.L., and Hines, J.E. (2006). Occupancy estimation and modelling. Inferring Patterns and Dynamics of Species Occurrence. Academic Press, London.

Netler, M., and Mitasova, H. (2008). Open source GIS a Grass GIS approach. Springer, New York.

O'Dowd, D.J., and Lake, P.S. (1990). Red crabs in rain forests, Christmas Island: differential herbivory of seedlings. Oikos 48(3): 289-292.

O'Dowd, D.J., and Lake, P.S. (1991). Red crabs in rain forests, Christmas Island: removal and fate of fruits and seeds. Journal of Tropical Ecology 7(1): 113-122.

Royle, J.A., and Dorazio, R.M. (2008). Hierarchical modelling and inference in ecology. Academic Press, London.

$\mathrm{R}$ Development Core Team (2007). R: A language and environment for statistical computing. R Foundation for Statistical Computing, Vienna.

MANUSCRIPT RECEIVED 18 AUGUST 2011; ACCEPTED 17 NOVEMBER 2011. 